\title{
Considerations in Orbital Reconstruction for the Oncologic Surgeon: Critical versus Optimal Objectives
}

\author{
Alex T. Legocki ${ }^{1} \quad$ Brett A. Miles ${ }^{1}$ \\ ${ }^{1}$ Department of Otolaryngology-Head and Neck Surgery, Icahn \\ School of Medicine, Mount Sinai Health System, New York, \\ United States
}

\begin{abstract}
Address for correspondence Brett A. Miles, MD, DDS, FACS, Department of Otolaryngology-Head and Neck Surgery, Mount Sinai Medical Center, Annenberg 10th Floor, One Gustave L. Levy Place, Box 1189, New York, NY 10029-3136, United States (e-mail: Brett.miles@mountsinai.org).
\end{abstract}

\begin{abstract}
Keywords

- orbit

- globe

- reconstruction

- midface

- exenteration

- flap

- approach

- planning

Background Orbital reconstruction following oncologic midface resection is uniquely challenging, and makes critical contributions to patient aesthetics, function, and identity. Approach is largely dependent on surgeon and patient preferences, and there exists no consensus on defect characterization.

Objective The goal of the study is to provide a mental framework for the reconstructive oncologic surgeon to use as a foundation during his or her approach to the orbit. Design The design of the study is based on the review of current literature and expert opinion.

Conclusions Critical versus optimal objectives must be set in orbital reconstruction, and a systematic approach should be followed. We approach orbital reconstruction by first deciding whether globe-sparing surgery is possible, or if orbital exenteration will be necessary. We then set critical and optimal objectives for our chosen pathway. Critical goals in globe-sparing reconstruction include maintaining orbital volume and preserving visual function, and an optimal goal includes preservation of the nasolacrimal system. Critical goals in orbital exenteration include obliterating the defect, sealing the skull base and nasal cavities, and allowing eye protection to be worn over the contralateral eye postoperatively. Optimal goals in exenteration include preparation for prosthetics, volume and bony replacement, eyelid-sparing technique, and consideration of postoperative radiation.
\end{abstract}

\section{Introduction}

Reconstruction for orbital involvement during midface oncologic surgery is uniquely complex. The function of the eye is extremely critical to maintaining quality of life, and appropriate reconstruction makes this possible if planned correctly. In addition, the eye makes a significant contribution to personal identity and aesthetics, and this deserves appropriate consideration as well.

The orbit is often considered the most challenging part of midface reconstruction. The approach will vary based primarily on the resultant defect, as well as the need for adjuvant therapy. In cases when orbital exenteration is required, reconstructive decisions will often be driven by available

published online Augus 29, 2019
DOI https://doi.org/

10.1055/s-0039-1696624

ISSN 0970-0358. resources and by the patient's desire to wear a prosthesis or eye patch. If the globe is preserved, profound aesthetic and functional deficits are common, including enophthalmos, hypophthalmos, lid malposition, epiphora, and diplopia. Secondary procedures are often unsuccessful in correcting structural derangements, especially if adjuvant radiation is administered, and so care must be taken to select the optimal initial reconstructive option, when possible. ${ }^{2}$ During the surgical planning process, it is critical to take a systematic approach, but currently no orbit-centered framework exists for midface oncologic reconstructions.

The current literature is scattered with various approaches to maxillary and midface reconstruction based on the planned maxillary defect. Defect classification systems such

(C)2019 Association of Plastic Surgeons of India

\section{License terms}

(1) $\Theta \circledast$ 
as those proposed by Kesting, Okay, Brown, and Cordeiro address various volumes and orientations of midface defects, with consideration of planned prostheses, obturators, and implants. The orbit is considered at some level in each classification system; however, focus is placed on the midface defect as a whole. A structured approach to the orbit itself is absent. While many of these approaches can be helpful, the variety of situations is innumerable and often complex algorithms and classification systems can cloud the situation. In addition, there is no consensus among these defect classifications and consequently there is no standardized approach to reconstruction..$^{3-9}$ Reconstruction of orbital defects is largely dependent on the surgeon's experience, though generally acceptable outcomes have been reached using locoregional (e.g., temporalis muscle) or small free flaps (e.g., radial forearm) for defects confined to the orbit and small portions of orbital rim, and larger free flaps (e.g., anterolateral thigh, rectus abdominis, scapula, and latissimus dorsi) for larger, more complex midface defects. . $^{3,9-15}$

We provide both critical and optimal objectives to consider in orbital reconstruction, to provide an additional conceptual framework to the reconstructive surgeon (-Fig. 1). In general, the most critical inflection point in planning for orbital reconstruction is relatively obvious, namely whether preservation of the globe will be pursued. In the first section we address reconstructive planning in situations with globe preservation, followed by considerations in cases requiring orbital exenteration.

\section{Globe Preservation}

A variety of proposed indications exist for orbital exenteration in maxillary and sinonasal malignancies, though there exists no steadfast rule and the complexity of the decision to remove the eye is beyond the scope of this article. Generally, exenteration is performed if the malignancy invades the periorbita, extraocular muscles, or orbital apex, and certainly in cases where vision loss has already occurred. It should be noted; however, that with improved imaging, neoadjuvant and adjuvant therapy, and the focus on function and quality of life, the indications for orbital exenteration have diminished in the current era. ${ }^{2,16-20}$ If oncologic outcomes are similar and orbital preservation is considered, then the reconstructive goals can be divided into critical objectives and optimal objectives.

\section{Globe Preservation: Critical Objectives}

Critical objectives during globe-sparing orbital reconstruction are (1) preservation of orbital volume and (2) preservation of visual function. These critical factors mirror the fundamental principles of orbital trauma reconstruction. ${ }^{21}$ Maintaining orbital volume is principal, as it will translate into appropriate globe position and reduce the risk of enophthalmos, ectropion, diplopia, and extraocular muscle dysfunction. This should be achieved with rigid support (as opposed to soft tissue).2,22,23 Accuracy here requires a preoperative understanding of the planned defect and adequate graft or implant geometry to replace all resected orbit walls. Cordeiro simplifies the orbit into a six-walled structure that attributes purpose to each side, separating it from adjacent spaces, such as the maxillary sinus and nasal cavity. ${ }^{3}$ This schema holds true on a fundamental level, but efforts to replace the defect with an identically-contoured piece should be attempted in all cases of globe-sparing reconstruction. The subtle curves of the orbital floor must be considered, and volume cannot be reduced to a simple cone as is often the perception. Respecting these anatomic subtleties will result in maximal aesthetic and functional preservation by reproducing

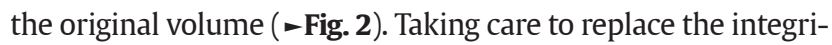
ty of the floor and walls will reduce the risk of entrapment and resulting diplopia. Again, all efforts are aimed to optimize the primary reconstruction, as structural derangements are often refractory to late secondary repair. ${ }^{2}$

Modern methods include aiding classic reconstructive techniques with computer-assisted design (CAD) software. Three-dimensional (3D) renderings of the bony midface are created based on computed tomography (CT) images. These can be used to fabricate stereolithographic models that are ideal for surgical planning and preoperative plate bending. ${ }^{24,25}$ In addition; commercially-available virtual surgical planning (VSP) services can render a computer image of the planned defect, and then overlay a planned implant or the surgeon's planned bony free flap. In the latter, cutting vectors are calculated to yield the desired donor shape and size. High-end printing techniques can then produce contoured implantable plates or osteotomies of autologous bone grafts, which have resulted in more precision in orbital structure and volume preservation. ${ }^{26}$

The second critical objective-vision preservation-will be influenced somewhat by orbital volume changes, but is also largely impacted by eyelid position and function as well as avoiding disruption of the extraocular muscles. The need to avoid extraocular muscle entrapment, damage, and denervation is obvious. Maintaining essential eyelid position and function can be a considerable challenge depending on the defect and adjuvant radiation. The aim is primarily to avoid globe exposure; however, other consequences of compromised eyelid function include reduced quality of life and poor aesthetics. Globe exposure can result from ectropion, midface ptosis, eyelid retraction, and facial nerve palsy, and the most critical consequence is impaired vision due to blepharitis, conjunctivitis, exposure keratitis, and dry eye. ${ }^{2}$ Ectropion is seen often in orbital reconstruction-in up to $70 \%$ of cases-and is also a common cause of poor aesthetic outcome that frequently requires surgical correction., ${ }^{2,39}$ Primary techniques such as lower lid canthopexy, frost sutures, and corneal protection should be employed during the reconstruction to avoid globe exposure (-Fig. 3). In cases when lid function is suboptimal, repair of residual deficits should be attempted. Traditional secondary techniques commonly include canthopexy or canthoplasty for ectropion, face-lift and implant or graft placement for midface ptosis and eyelid retraction, and gold weight implants for facial nerve paralysis. ${ }^{27}$ These measures are designed to protect the cornea, thereby avoiding long-term corneal keratitis and vision impairment, but all are more challenging in the setting of adjuvant radiation $^{28,29}$ ( - Fig. 4). In addition to surgical techniques, careful analysis of postoperative imaging to ensure appropriate implant position, as well as appropriate clinical management 


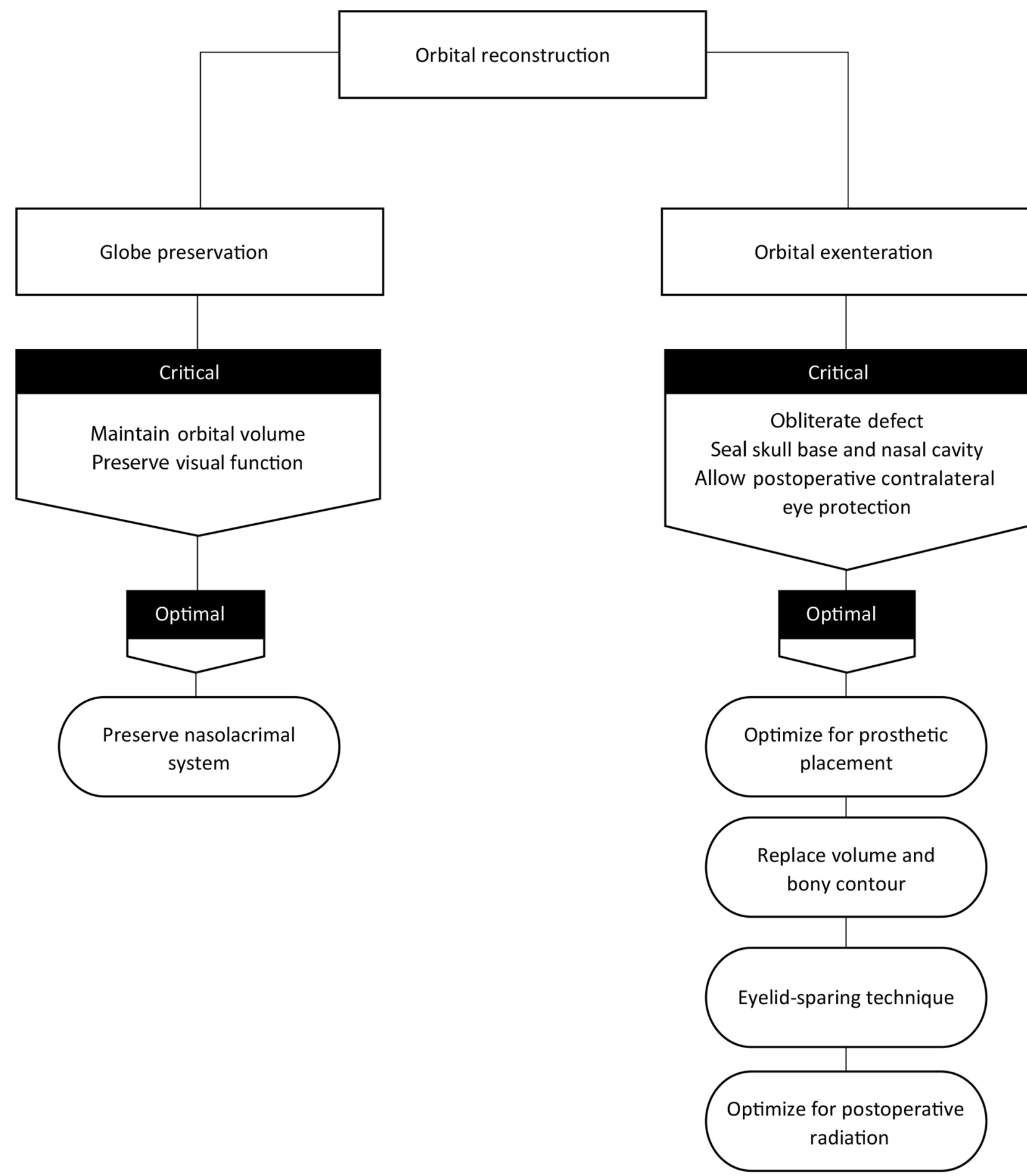

Fig. 1 Algorithmic approach to orbital reconstruction. A systematic consideration of the critical and optimal goals of orbital reconstruction can improve the surgeon's preoperative planning. All aspects should be discussed with the patient in the preoperative setting.

of postoperative complications such as orbital hematoma are important to achieve these critical objectives.

\section{Globe Preservation: Optimal Objectives}

Optimal objectives of orbital reconstruction are those objectives that lead to improved outcomes, but are not critical to the function of the orbit and eye. The optimal goal to be considered in globe-sparing reconstruction is the preservation of the nasolacrimal system (NLS). Midface resection with transection of the NLS results in rates of persistent epiphora as high as 63\%. Various methods of NLS management exist, including simple transection, transection with several methods of stenting, and marsupialization with or without stenting. The approach is dependent on surgeon comfort, and no option has shown to be consistently superior. ${ }^{1,2,30}$ Exploring the spectrum of NLS 


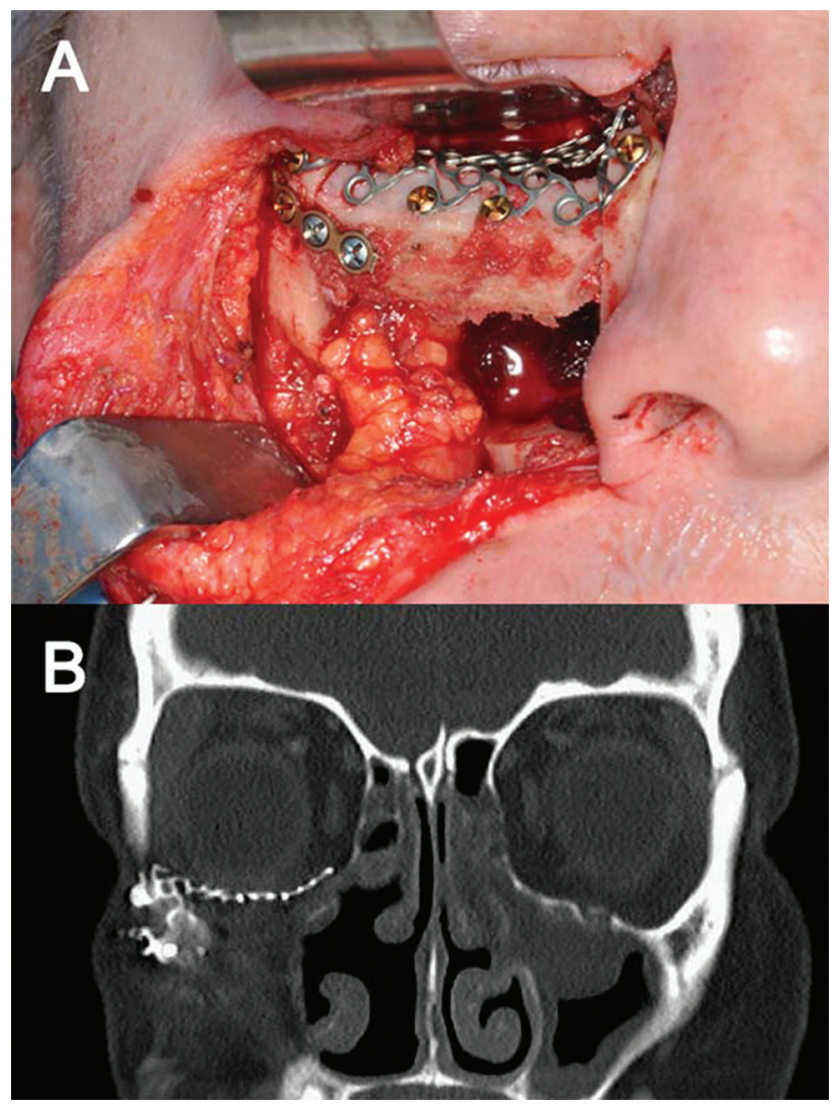

Fig. 2 Orbital volume should be replaced by replicating the natural contour of the resected bone. (A) Titanium mesh plating with a concave form to replace the orbital floor. (B) Axial cut of CT facial bones showing curvature of implanted plate with an attempt to match the contralateral orbital floor. CT, computed tomography.

management options is beyond the scope of this report; however, we stress the importance of avoiding unnecessary trauma to the NLS in the setting of globe-sparing reconstruction. Knowledge of the NLS anatomy and a carefully planned resection around its components will reduce unnecessary revisions. In all cases of prolonged epiphora, dacryocystorhinostomy should be performed. Involving an ophthalmologist early in the treatment planning phase can improve outcomes and should be standard of care when feasible.

\section{Orbital Exenteration}

Interestingly, once the decision has been made that orbital exenteration is indicated, many surgeons will limit the reconstructive planning to techniques designed to "fill the hole" and associate achieving this goal with "successful reconstruction." This type of thinking is somewhat limited, and outcomes can be improved by developing an understanding of the patient's preferences. If the globe must be sacrificed, the preoperative planning begins by discussing the reconstructive options with the patient, along with an evaluation of available resources. Certainly, while a patient may wish to have an implant-retained orbital prosthesis instead of a skin paddle reconstruction or eye patch, if there are insufficient resources then the plan will require alteration. Involving the patient in this decision-making

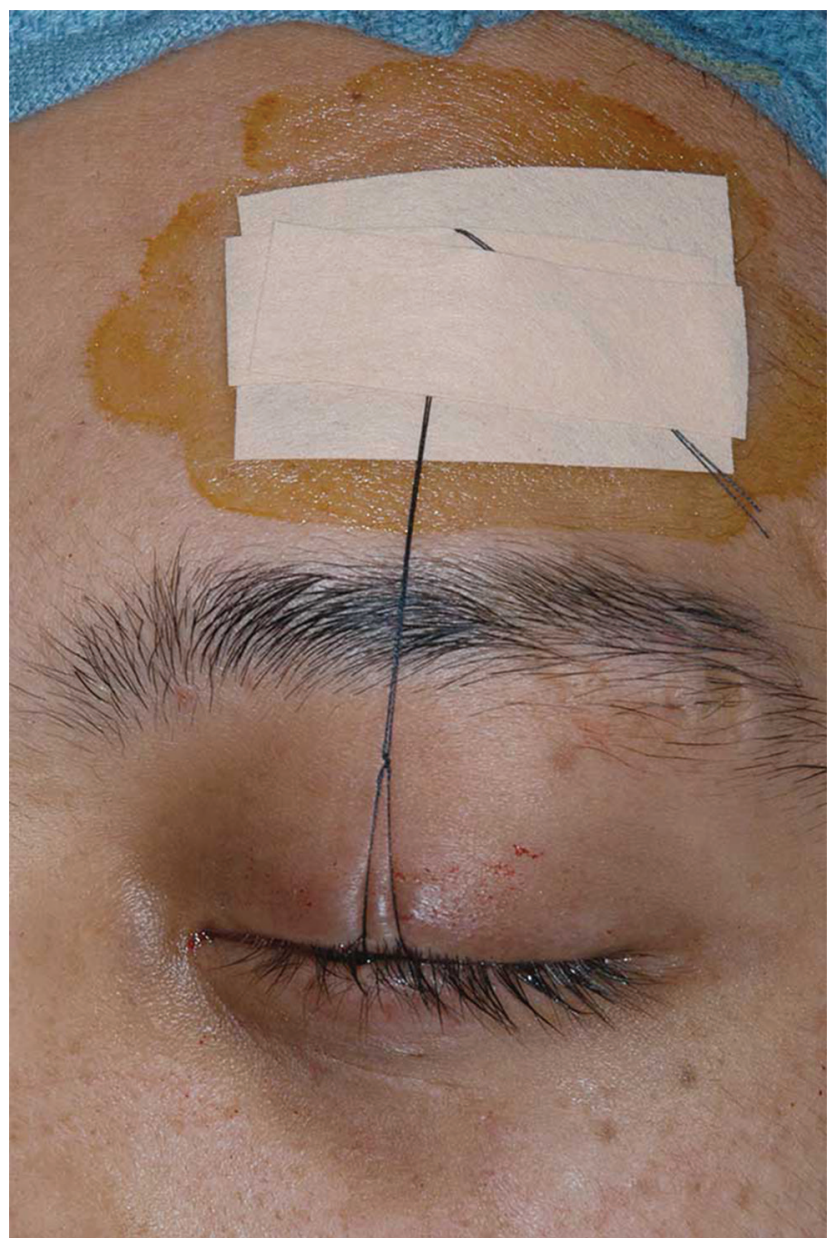

Fig. 3 Frost suture placed intraoperatively to avoid post-operative ectropion of the lower lid.

process will result in improved satisfaction, regardless of which aesthetic goal is chosen.

\section{Orbital Exenteration: Critical Objectives}

Ablations requiring orbital exenteration inherently tend to be larger defects; however, given that the eye is no longer viable, the reconstruction-though perhaps technically challenging-has relatively simple tenets. Two objectives that we consider to be critical in such a reconstruction are (1) to obliterate the defect, and (2) to seal the skull base and nasal cavity if they are violated. The aim here is to prevent orbito-nasal or orbito-sinus fistulas, cerebrospinal fluid (CSF) leaks, dural exposure, and meningitis ${ }^{31}$ (-Fig. 5). If the aforementioned complications are a concern or the defect is large, free tissue transfer of appropriate bulk-such as an anterolateral thigh, rectus abdominis, latissimus dorsi, serratus anterior, or scapula-serves as an ideal reconstructive option to provide volume. Similarly for those patients that will require postoperative radiation, these flaps offer robust vascularized tissue to withstand volume changes. In addition, using such reliable reconstruction techniques prevents long-term complications such as exposed bone, CSF leak, and other issues that may delay or complicate adjuvant therapy. 


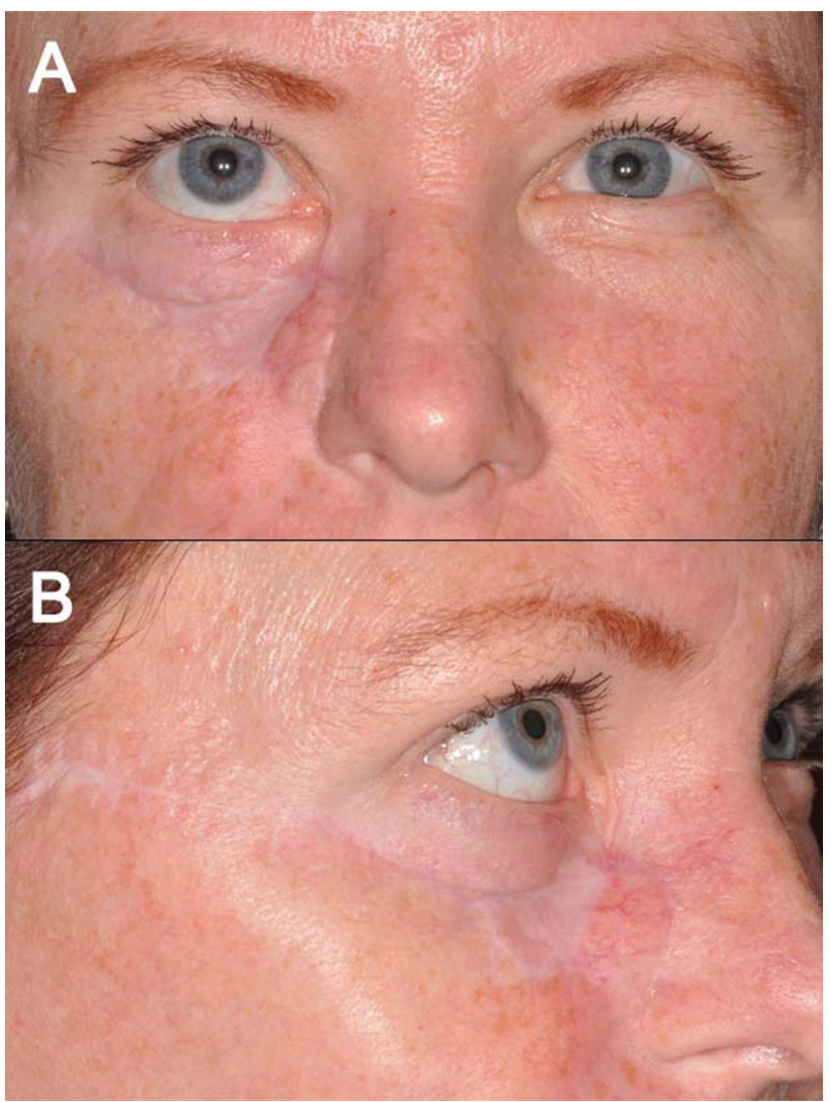

Fig. 4 Frontal view (A) and profile view (B) of right lower lid ptosis following reconstruction and adjuvant radiation. Note the contracted skin at the site of reconstruction, which makes for a challenging secondary repair.

A third, often-ignored critical objective of reconstructive surgery after orbital exenteration is (3) protection of the remaining eye. Bulky reconstructions that prevent the patient from wearing protective glasses should be avoided at all costs, as an injury to a patient's only remaining eye is catastrophic from a functional and quality-of-life standpoint. Reconstructive surgeons would be wise to remember this fact when formulating a reconstructive plan.

\section{Orbital Exenteration: Optimal Objectives}

The optimal outcome of globe-sacrificing orbital reconstruction is to create an appropriate foundation for the patient's preferred aesthetic goals. For this purpose, consideration of prosthetics, bone and volume replacement, eyelid-sparing reconstruction, and postoperative radiation are key.

While many patients initially opt for an orbital prosthesis, data indicates that these devices are associated with inconsistent use, and some studies suggest that most patients will revert to an eye patch. That being said, successful prosthetic rehabilitation has been associated with excellent aesthetics and socially-acceptable outcomes. ${ }^{32-34}$ When planning for an orbital prosthesis, a concave reconstructed orbit is imperative to allow for sufficient room for prosthetic placement. This can be achieved by allowing the cavity to granulate, or by placing a smaller-volume graft into the defect. The former has the disadvantage of

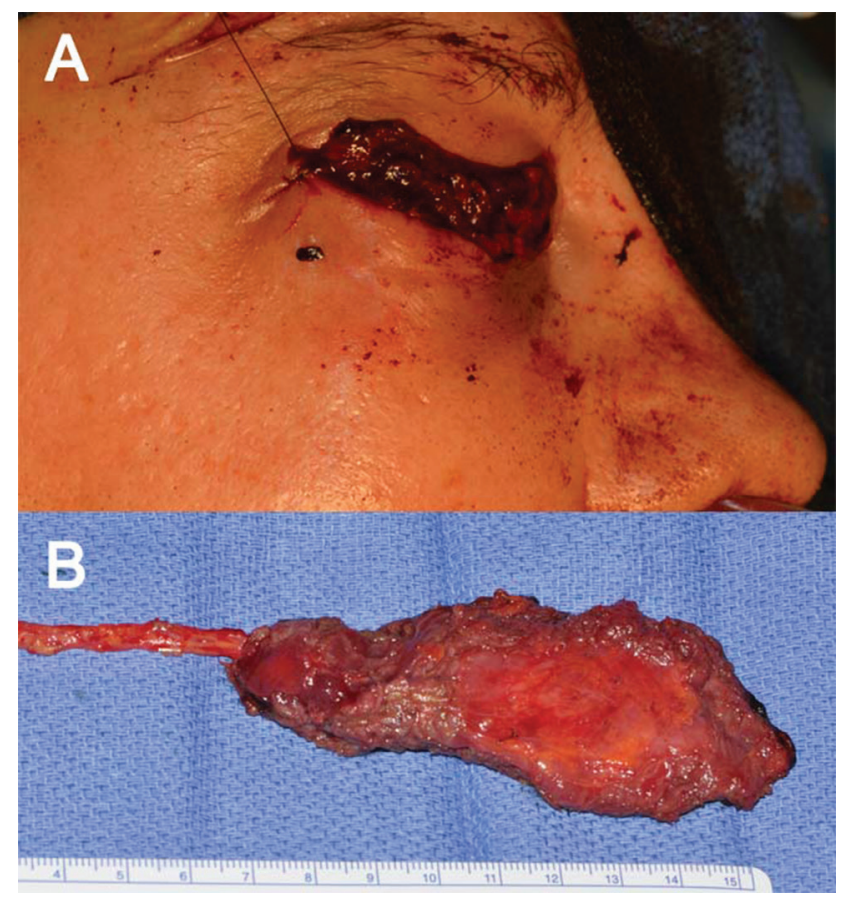

Fig. 5 Reconstruction following orbital exenteration. (A) Free tissue transfer allows for volume replacement and defect obliteration. Excess bulk is necessary in the setting of adjuvant radiation. (B) The serratus anterior muscle flap can provide mid-sized soft tissue replacement.

causing periorbital tissue contraction and takes more time to heal, and is not often considered when addressing a larger defect that may include orbital rim and surrounding structures. ${ }^{35}$ In addition, this technique is less able to withstand adjuvant radiation and should be avoided in these situations. The latter reconstruction can generally be achieved with split-thickness and full-thickness skin grafts, pedicled temporoparietal fascia or temporalis muscle flaps, or fasciocutaneous or osseocutaneous radial forearm free flaps if limited to the orbit and a small portion of the orbital rim..$^{14,34,36}$ Ultimately, the optimal postoperative situation is a concave orbital cavity lined with thin skin, preferably with an excellent color match to allow for optimal prosthesis camouflage (-Fig. 6).

In cases when prosthetic rehabilitation is not planned, or in larger, extended resections (i.e., those involving significant bone and/or surrounding tissues), attention should be aimed at the replacement of volume and reconstruction of the orbital rim for aesthetic purposes. The malar eminence and orbital rim provide a natural facial contour that should be restored. This volume is best replaced with a free flap to obliterate the deeper defect and provide soft tissue support. If the rim remains intact, the myocutaneous anterolateral thigh and rectus abdominis will adequately provide this volume. For rim replacement, the osseocutaneous scapula flap allows for ample vascularized bone and soft tissue, ${ }^{14}$ although bulk can often be an issue with this option. As noted earlier, excessive bulk should be avoided to allow for protective glasses to be worn avoiding injury to the remaining eye.

In many cases, eyelid-sparing surgery should be considered. As previously mentioned, the eyelid is difficult to 


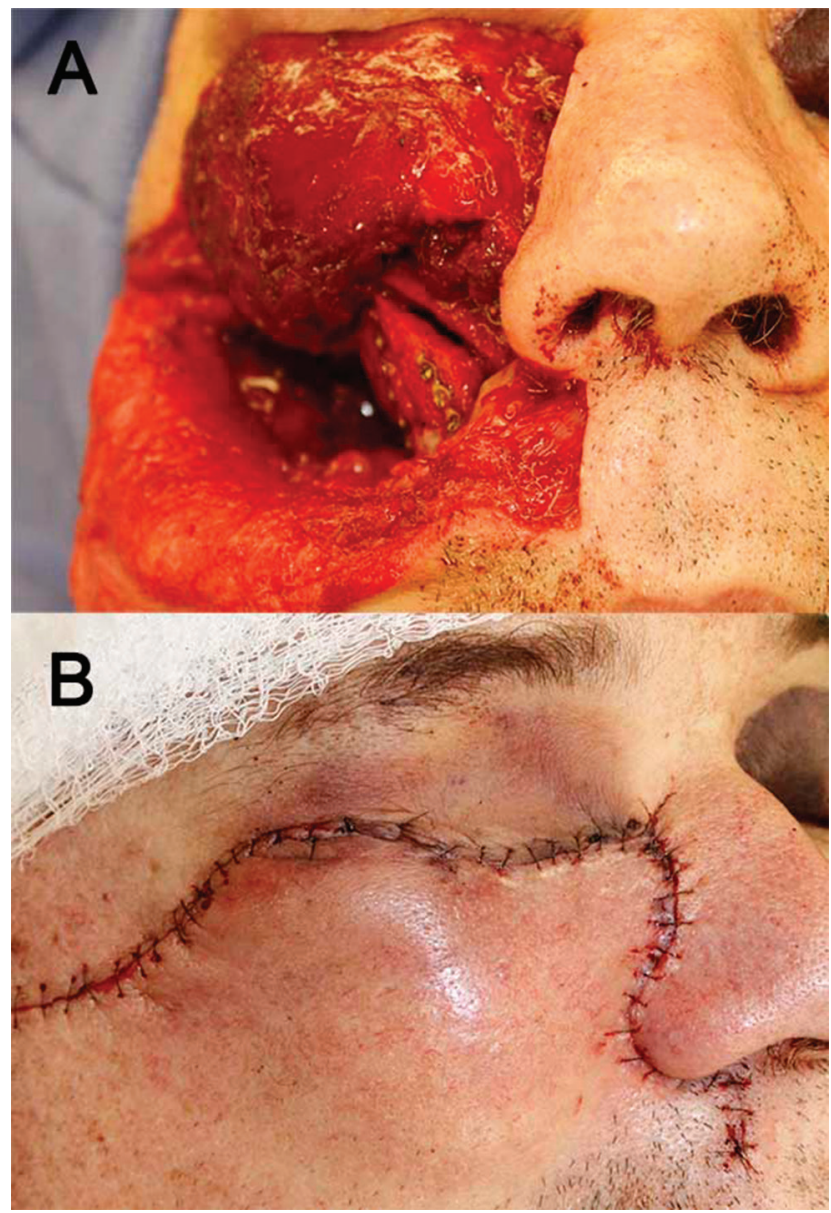

Fig. 6 Reconstruction following orbital exenteration, in preparation for prosthesis. (A) Scapula free flap reconstruction provides bony structure to the orbital rim. (B) Excellent skin color matching and limited bulk allow for optimal prosthesis use.

reconstruct without significant distortion, and serves as a key aesthetic element of the midface. Following deep tissue volume replacement, it can be sutured to the lower lid to provide excellent color matching and preserve contours of facial expression. Some data suggests lower rates of eye patches worn by patients with lid-sparing procedures. In addition, eyelid-sparing reconstruction is associated with a significantly faster recovery ${ }^{34,37}$ ( - Fig. 7).

If the patient's treatment plan includes-or may includepostoperative radiation, there are additional considerations. Supplemental soft tissue overlying osseous prominences may be necessary to account for postradiation reduction in flap volume and to prevent bony exposure that carries risk of osteoradionecrosis. The same principle holds true for metallic or alloplastic reconstructive implants. The degree of volume loss varies between studies and flap types, generally ranging from 20 to $40 \%{ }^{38-40}$ In addition, bone grafts should be vascularized when feasible to reduce the likelihood of resorption and osteoradionecrosis. Soft tissue fibrosis and contraction is unpredictable, and the option for a future prosthesis should be reserved and accounted for during reconstruction.

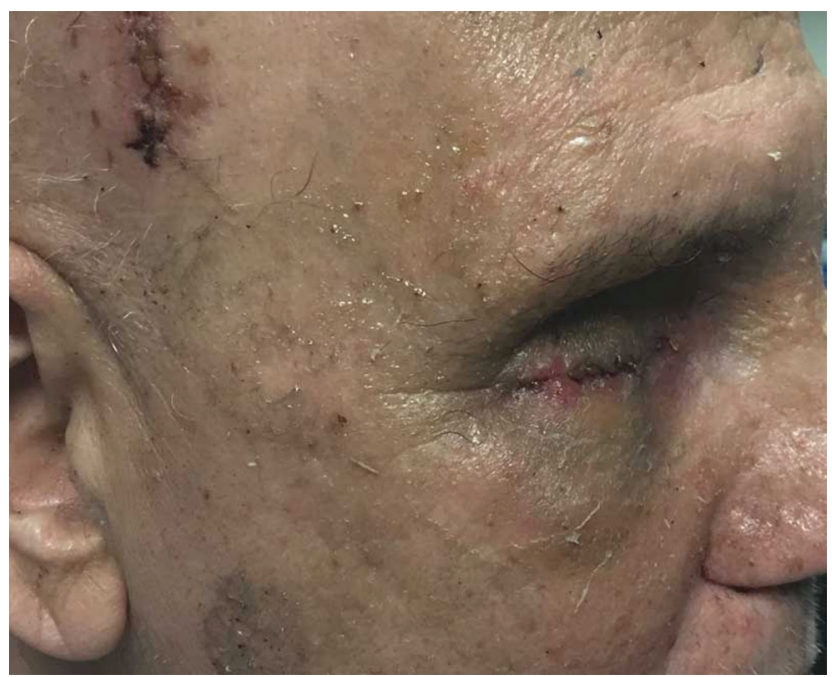

Fig. 7 Eyelid-sparing surgical technique aims to replace orbital volume to match the contralateral eye. The upper and lower lids are sutured to provide excellent color matching and some preservation of facial expression.

\section{Conclusion}

In this article, we outline critical and optimal objectives in orbital reconstruction in the setting of midface oncologic resection, to clarify the goals of surgical planning for the reconstructive surgeon. If the orbit is preserved, critical objectives include maintaining orbital volume and visual function, and optimal objectives include preserving the nasolacrimal system. If the globe is sacrificed, critical objectives include defect obliteration, skull base and nasal cavity closure, and adequate protection of the remaining eye postoperatively. Optimal objectives include the following aesthetic considerations: preparation for prosthetics, bone and volume replacement, eyelid-sparing technique, and preparation for postoperative radiation.

Above all, it is critical to include the patient in the entire decision-making process. Realistic patient expectations can only come from a clear understanding of the reconstructive options, available resources, and short- and long-term aesthetic and functional consequences. Likewise, a surgeon's true understanding of each patient's goals is paramount to achieve optimal outcomes in a surgical arena that lacks a standardized approach.

\section{Presented at}

Challenges in Orbital Reconstruction; presented at the AOCMF Advances in Maxillofacial Reconstruction and Rehabilitation Symposium at the World Oral Cancer Congress of the International Academy of Oral Oncology, Bangalore, India, May 17, 2017.

\section{Financial Support}

None declared.

\section{Conflict of Interest}

None declared. 


\section{References}

1 Habib R, Har-El G. Management of the lacrimal system during maxillectomy. Am J Rhinol 2004;18(6):367-370

2 Imola MJ, Schramm VL, Jr. Orbital preservation in surgical management of sinonasal malignancy. Laryngoscope 2002;112(8 Pt 1):1357-1365

3 Cordeiro PG, Santamaria E. A classification system and algorithm for reconstruction of maxillectomy and midfacial defects. Plast Reconstr Surg 2000;105(7): 2331-2346,

4 Brown JS, Rogers SN, McNally DN, Boyle M. A modified classification for the maxillectomy defect. Head Neck 2000;22(1):17-26

5 Brown JS, Shaw RJ. Reconstruction of the maxilla and midface: introducing a new classification. Lancet Oncol 2010;11(10):1001-1008

6 Okay DJ, Genden E, Buchbinder D, Urken M. Prosthodontic guidelines for surgical reconstruction of the maxilla: a classification system of defects. J Prosthet Dent 2001;86(4):352-363

7 Chang EI, Hanasono MM. State-of-the-art reconstruction of midface and facial deformities. J Surg Oncol 2016;113(8):962-970

8 Kesting MR, Koerdt S, Rommel N, et al. Classification of orbital exenteration and reconstruction. J Craniomaxillofac Surg 2017;45(4):467-473

9 Cordeiro PG, Chen CM. A 15-year review of midface reconstruction after total and subtotal maxillectomy: part I. Algorithm and outcomes. Plast Reconstr Surg 2012;129(1):124-136

10 Mücke T, Hölzle F, Loeffelbein DJ, et al. Maxillary reconstruction using microvascular free flaps. Oral Surg Oral Med Oral Pathol Oral Radiol Endod 2011;111(1):51-57

11 Spanio di Spilimbergo S, Nordera P, Mardini S, et al. Pedicled temporalis muscle flap for craniofacial reconstruction: a 35-year clinical experience with 366 flaps. Plast Reconstr Surg 2017;139(2):468e-476e

12 Simsek T, Engin MS, Yildirim K, Kodalak EA, Demir A. Reconstruction of extensive orbital exenteration defects using an anterolateral thigh/vastus lateralis chimeric flap. J Craniofac Surg 2017;28(3):638-642

13 Khan MN, Rodriguez LG, Pool CD, et al. The versatility of the serratus anterior free flap in head and neck reconstruction. Laryngoscope 2017;127(3):568-573

14 Chepeha DB, Wang SJ, Marentette LJ, et al. Restoration of the orbital aesthetic subunit in complex midface defects. Laryngoscope 2004;114(10):1706-1713

15 Miles BA, Gilbert RW. Maxillary reconstruction with the scapular angle osteomyogenous free flap. Arch Otolaryngol Head Neck Surg 2011;137(11):1130-1135

16 Dulguerov P, Jacobsen MS, Allal AS, Lehmann W, Calcaterra T. Nasal and paranasal sinus carcinoma: are we making progress? A series of 220 patients and a systematic review. Cancer 2001;92(12):3012-3029

17 Carrau RL, Segas J, Nuss DW, et al. Squamous cell carcinoma of the sinonasal tract invading the orbit. Laryngoscope 1999;109(2 Pt 1):230-235

18 Sakashita T, Hayashi R, Homma A, et al. Multi-institutional retrospective study for the evaluation of ocular function-preservation rates in maxillary sinus squamous cell carcinomas with orbital invasion. Head Neck 2015;37(4):537-542

19 Lisan Q, Kolb F, Temam S, Tao Y, Janot F, Moya-Plana A. Management of orbital invasion in sinonasal malignancies. Head Neck 2016;38(11):1650-1656

20 Howard DJ, Lund VJ, Wei WI. Craniofacial resection for tumors of the nasal cavity and paranasal sinuses: a 25-year experience. Head Neck 2006;28(10):867-873
21 Hollier LH, Jr. Sharabi SE, Koshy JC, Stal S. Facial trauma: general principles of management. J Craniofac Surg 2010;21(4):1051-1053

22 Gilbard SM, Mafee MF, Lagouros PA, Langer BG. Orbital blowout fractures. The prognostic significance of computed tomography. Ophthalmology 1985;92(11):1523-1528

23 Raskin EM, Millman AL, Lubkin V. della Rocca RC, Lisman RD, Maher EA. Prediction of late enophthalmos by volumetric analysis of orbital fractures. Ophthal. Plast Reconstr Surg 1998;14(1):19-26

24 Rengier F, Mehndiratta A, von Tengg-Kobligk H, et al. 3D printing based on imaging data: review of medical applications. Int J CARS 2010;5(4):335-341

25 Azuma M, Yanagawa T, Ishibashi-Kanno N, et al. Mandibular reconstruction using plates prebent to fit rapid prototyping 3-dimensional printing models ameliorates contour deformity. Head Face Med 2014;10:45

26 Zimmerer RM, Ellis E, III. Aniceto GS, etal. A prospective multicenter study to compare the precision of posttraumatic internal orbital reconstruction with standard preformed and individualized orbital implants. J Craniomaxillofac Surg 2016;44(9):1485-1497

27 Moskowitz BK, Patel AD, Pearson JM. Aesthetic and functional management of eyelid and orbital reconstruction. Facial Plast Surg 2008;24(1):69-77

28 Chi JJ. Management of the eye in facial paralysis. Facial Plast Surg Clin North Am 2016;24(1):21-28

$29 \mathrm{Kim} \mathrm{KH}$, Baek JS, Lee S, et al. Causes and surgical outcomes of lower eyelid retraction. Korean J Ophthalmol 2017;31(4):290-298

30 Yeo NK, Wang JH, Chung YS, Jang YJ, Lee BJ. Contributing factors to prevent prolonged epiphora after maxillectomy. Arch Otolaryngol Head Neck Surg 2010;136(3):229-233

31 Bartley GB, Garrity JA, Waller RR, Henderson JW, Ilstrup DM. Orbital exenteration at the Mayo Clinic. 1967-1986. Ophthalmology 1989;96(4):468-473

32 Kuiper JJ, Zimmerman MB, Pagedar NA, Carter KD, Allen RC, Shriver EM. Perception of patient appearance following various methods of reconstruction after orbital exenteration. Orbit 2016;35(4):187-192

33 Ben Simon GJ, Schwarcz RM, Douglas R, Fiaschetti D, McCann JD, Goldberg RA. Orbital exenteration: one size does not fit all. Am J Ophthalmol 2005;139(1):11-17

34 Goldberg RA, Kim JW, Shorr N. Orbital exenteration: results of an individualized approach. Ophthal. Plast Reconstr Surg 2003;19(3):229-236

35 Putterman AM. Orbital exenteration with spontaneous granulation. Arch Ophthalmol 1986;104(1):139-140

36 Hanasono MM, Lee JC, Yang JS, Skoracki RJ, Reece GP, Esmaeli B. An algorithmic approach to reconstructive surgery and prosthetic rehabilitation after orbital exenteration. Plast Reconstr Surg 2009;123(1):98-105

37 Shields JA, Shields CL, Demirci H, Honavar SG, Singh AD. Experience with eyelid-sparing orbital exenteration: the 2000 Tullos 0 . Coston Lecture. Ophthal Plast Reconstr Surg 2001;17(5):355-361

38 Cho KJ, Joo YH, Sun DI, Kim MS. Perioperative clinical factors affecting volume changes of reconstructed flaps in head and neck cancer patients: free versus regional flaps. Eur Arch Otorhinolaryngol 2011;268(7):1061-1065

39 Fujioka M, Masuda K, Imamura Y. Fatty tissue atrophy of free flap used for head and neck reconstruction. Microsurgery 2011;31(1):32-35

40 Bittermann G, Thönissen $P$, Poxleitner $P$, Zimmerer $R$, Vach K, Metzger MC. Microvascular transplants in head and neck reconstruction: 3D evaluation of volume loss. J Craniomaxillofac Surg 2015;43(8):1319-1324 